\title{
Glycine Interaction with Carbon Nanotubes: An ab Initio Study
}

\author{
Andreas Mavrandonakis, ${ }^{\ddagger}$ Stavros C. Farantos,,$+\dagger$ and George E. Froudakis $*, \$$ \\ Department of Chemistry, University of Crete, P.O. Box 1470, Iraklion 714 09, Crete, Greece, and \\ Institute of Electronic Structure and Laser, Foundation for Research and Technology-Hellas, P.O. Box 1527, \\ Iraklion 711 10, Crete, Greece
}

Received: December 14, 2005

\begin{abstract}
The interaction of the glycine radical on the side walls of both armchair and zigzag single walled carbon nanotubes is investigated by density functional theory. It is found that the interaction potential of the $\mathrm{N}$-centered glycine radical with the tubes has a minimum of 16.9 (armchair) and 20.2 (zigzag) $\mathrm{kcal} / \mathrm{mol}$ with respect to the dissociation products. In contrast, the $\mathrm{C}$-centered radical, which is $22.7 \mathrm{kcal} / \mathrm{mol}$ lower in energy than the $\mathrm{N}$-centered radical, does not form stable complexes with both types of carbon nanotubes.
\end{abstract}

\section{Introduction}

Since their discovery in 1991 carbon nanotubes $^{1}$ (CNTs) have attracted considerable interest in nanotechnology due to their unique shape. Their outstanding mechanical and electronic properties have made them promising materials for new applications such as nanoelectronic devices, fuel storage materials and energy capacitors. ${ }^{2,3}$ However, few applications have so far been reported in biology. The selective opening and closing ${ }^{4}$ at the ends of nanotubes provide many opportunities of conducting experiments with biological macromolecules in nanoscale test tubes. The immobilization of oligonucleotides and proteins can be done either inside ${ }^{5}$ or outside of $^{6,7}$ the nanotubes, without losing their activity and immunological properties. ${ }^{8,9}$ A recent study has shown that the use of CNTs as liquid filled nanoparticles for drug delivery tool improves the bioavailability of the erythropoietin drug solution up to $11.5 \%$ compared to other drug adsorbents. ${ }^{10}$ This is an example of the potential applications of carbon nanotubes in biomedicine as drug or vaccine carriers and biomolecular recognition. ${ }^{11}$

Despite their technological and biological interest there is no detailed theoretical analysis of the interactions of CNTs and biological molecules such as amino acids. There are only reports about the addition of small organic molecules on CNTs, ${ }^{12,13}$ which do not clarify the nature of the interaction CNTs-amino acids. This is crucial because amino acids can serve as a first step in extending the system to peptides and proteins.

In this letter, we examine the "direct" interaction of glycine radical with the walls of a $(4,4)$ armchair and an $(8,0)$ zigzag CNT. The glycine radicals have been successfully produced in the gas-phase ${ }^{14}$ reaction of positively charged phenyl radicals with the neutral Glycine molecule as well as in liquid phase. ${ }^{15}$ Several other studies have reported the interaction of peptides or amino acids with functionalized CNTs, ${ }^{16}$ but to the best of our knowledge, this is the first study regarding the decoration of pure CNTs with amino acids.

\section{Computational Details}

To investigate the structural and electronic properties of CNTs decorated with the glycine radicals, we use density functional

\footnotetext{
* Corresponding author. Fax: +30 2810 393601; E-mail address: frudakis@chemistry.uoc.gr.

$\doteqdot$ Department of Chemistry.

$\dagger$ Institute of Electronic Structure and Laser.
}

theory (DFT). The problem that arises is how to perform an accurate calculation to a nanosized system without ending in a prohibitive large computation. A solution is the cluster model. A large enough part of an armchair $(4,4)$ and a zigzag $(8,0)$ CNT containing 64 carbon atoms (or 3 hexagon layers) was separated and treated as an individual system. The dangling bonds at the ends of the tubes were saturated by hydrogen atoms. The resolution of identity DFT (RI-DFT) ${ }^{18}$ as implemented in the TURBOMOLE ${ }^{17}$ program package in combination with the BLYP functional along with the SVP auxiliary basis set was employed for the geometry optimizations. All the structures discussed are fully optimized without any symmetry constraints.

\section{Results and Discussion}

As a first step of our investigation it was important to find the most stable isomers of the glycine radical and compare with previous works. Among the possible isomers are the ones from which one hydrogen atom is abstracted either from (a) the $\alpha \mathrm{C}$ atom, or (b) the $\mathrm{N}$-atom. It is found that the $\mathrm{C}$-centered radical is favored over the $\mathrm{N}$-centered one by $22.7 \mathrm{kcal} / \mathrm{mol}$ employing the BLYP functional. This is in reasonable agreement with the values of 18.5 and $19.9 \mathrm{kcal} / \mathrm{mol}$ reported in other ab initio studies. $^{14,19}$

In the second step we calculated the interaction of glycine radicals with the CNTs. Interestingly enough, we find that upon reaction with the tube walls, the glycine radical forms stable complexes when it reacts with the nitrogen atom and metastable conformations with $\alpha \mathrm{C}$-atom. All four structures, either $\mathrm{C}$ - or $\mathrm{N}$-centered glycine radicals with the $(4,4)$ and $(8,0)$ CNTs, are close in energy. The most stable configurations of the $\mathrm{C}$ - and $\mathrm{N}$-centered clusters are presented in Figure 1, along with their binding energies and relative stabilities.

According to a recent study by Radovic and co-workers ${ }^{20}$ carbon materials with zigzag graphene edges do not have the singlet state as their ground state. This is also verified by our results for the triplet state of the $(8,0) \mathrm{CNT}$, which is more favorable than the singlet by $7.1 \mathrm{kcal} / \mathrm{mol}$. Furthermore, this result was tested by conventional DFT calculations, as well as using the BP86 functional. The binding energies for all clusters bonded to the $(8,0) \mathrm{CNT}$ are calculated with respect to the triplet state of the tube.

Upon bonding with the glycine radical, the system has an odd number of electrons. So we have investigated both doublet 


\section{$\mathrm{N}$-approach}

\section{C-approach}
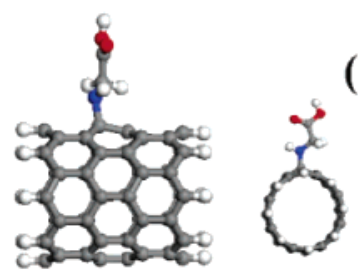

$(8,0)$

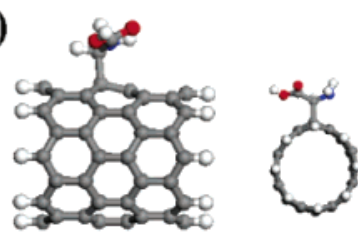

$0.0 \mathrm{kcal} / \mathrm{mol}$ B.E $=-20.2 \mathrm{kcal} / \mathrm{mol}$
$+0.5 \mathrm{kcal} / \mathrm{mol}$ B.E $=+3.5 \mathrm{kcal} / \mathrm{mol}$

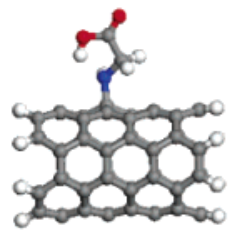

$+5.9 \mathrm{kcal} / \mathrm{mol}$

\section{B.E $=-\mathbf{1 6 . 9} \mathrm{kcal} / \mathrm{mol}$}

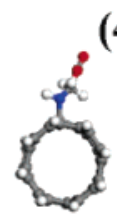

$(4,4)$

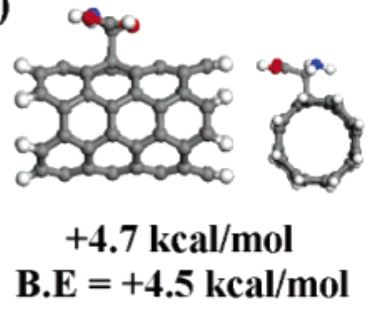

Figure 1. Optimized structures of the GLY radicals bonded to a $(4,4)$ and an $(8,0)$ CNT. The binding energies are computed with respect to the dissociation products (see text for details).

and quartet spin states of our isomers. For the $(4,4)$ case the quartet spin states are much higher in energy than the doublet, whereas for the $(8,0)$ case both spin states are close in energy and differ only by $2 \mathrm{kcal} / \mathrm{mol}$ at most.

The binding energies for the $\mathrm{N}$-centered radicals with respect to the dissociation products are calculated to be -16.9 for the $(4,4) \mathrm{CNT}$ and $-20.2 \mathrm{kcal} / \mathrm{mol}$ (quartet spin state) for the $(8,0)$ $\mathrm{CNT}$. In contrast, the $\mathrm{C}$-centered radicals have positive binding energies. This means that the addition of the $\mathrm{C}$-centered glycine radical to the tube walls is endothermic by $+3.5 \mathrm{kcal} / \mathrm{mol}$ for the $(8,0)$ case and +4.5 for the $(4,4) \mathrm{CNT}$. The N-centered cluster shows analogous structural characteristics to those reported by Zhao and co-workers. ${ }^{21}$ The authors have studied the chemical reactivity of CNTs to amidogen $\left(\cdot \mathrm{NH}_{2}\right)$, a species of smaller size but similar to the glycine radical $\left(\cdot \mathrm{NHCH}_{2}-\right.$ $\mathrm{COOH})$. They report a binding energy of amidogen to a $(5,5)$ CNT of $46 \mathrm{kcal} / \mathrm{mol}$, a value we were unable to reproduce. Instead, we have found that $\cdot \mathrm{NH}_{2}$ has a binding energy of 22 $\mathrm{kcal} / \mathrm{mol}$ to the walls of a $(5,5) \mathrm{CNT}$, which is similar to the binding energy of $\cdot \mathrm{NHCH}_{2} \mathrm{COOH}$.

The $\mathrm{N}$-centered clusters are almost isoenergetic with the C-centered. The small difference between these two isomers can be explained by the nature of the newly formed bond between the tube and glycine. In the $\mathrm{C}$-centered cluster, a single covalent $\mathrm{C}_{\text {glycine }}-\mathrm{C}_{\text {tube }}$ bond of $1.6 \AA$ is formed, with no significant electron transfer. In contrast, the $\mathrm{N}$-centered isomer has a $\mathrm{N}_{\text {glycine }}-\mathrm{C}_{\text {tube }}$ bond that is shorter (1.5 $\AA$ ) and shows a substantial electron transfer. The nitrogen atom is negatively charged by about $0.3|\mathrm{e}|$. Furthermore, considering the stability of these isomers, we have to take into account the stereochemical repulsions between the tube and the radical. In the $\mathrm{C}$-centered case, strong repulsions exist due to the backbone interaction of the glycine with the tube walls. In the $\mathrm{N}$-centered case, only the NH group is close enough to the tube walls and the strong repulsions are avoided.

We have also scanned the potential energy curves of the glycine addition and dissociation from the tube walls. We varied

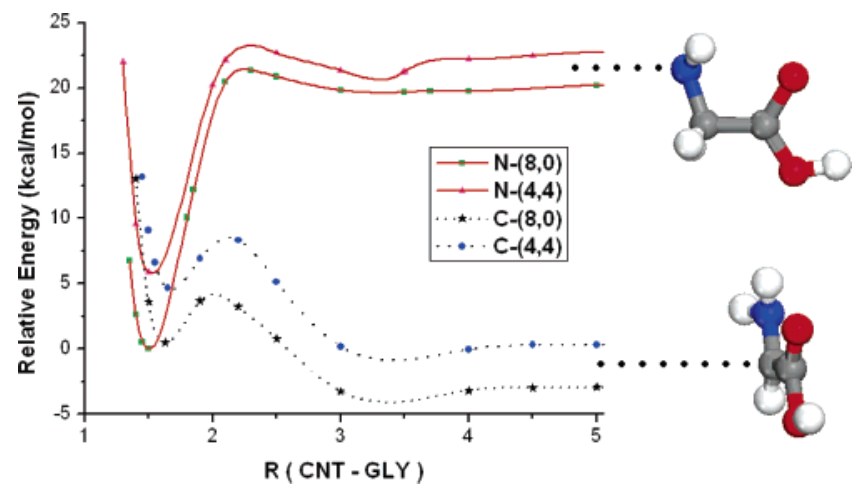

Figure 2. Dissociation energy curves of the GLY radicals from $(4,4)$ and $(8,0) \mathrm{CNT}$.

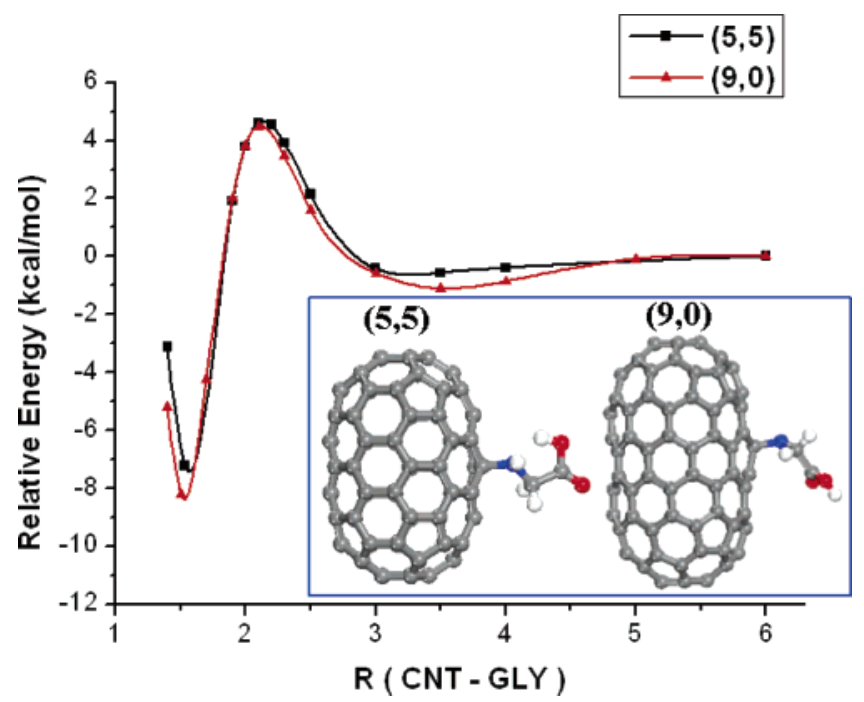

Figure 3. Dissociation energy curves of the GLY radicals from $(5,5)$ and $(9,0)$ capped CNTs. The inset picture shows the equilibrium structures of the GLY bonded to the capped CNTs.

the glycine $-\mathrm{C}_{\text {tube }}$ distance from 1.3 to $6 \AA$ and optimized all other degrees of freedom in every step for both $(4,4)$ and $(8,0)$ CNTs. In Figure 2, we clearly see two minima in the N-centered approach (solid lines). The first minimum at $1.5 \AA$ corresponds to the chemical adsorption of the $\mathrm{N}$-radical to the tube walls, whereas the second minimum at $3.5 \AA$ corresponds to the physical adsorption. The value for the physisorption energy is estimated to be about $1.5 \mathrm{kcal} / \mathrm{mol}$ for the armchair whereas it is only $0.5 \mathrm{kcal} / \mathrm{mol}$ for the zigzag case. A similar value has been reported for the physisorption energy and equilibrium distance of the ammonia molecule to CNT walls. ${ }^{22}$ Furthermore, for both tube cases the $\mathrm{N}$-centered addition reaction has to pass over a small energy barrier $(2 \mathrm{kcal} / \mathrm{mol})$. When the two adducts are formed either by approaching the $\mathrm{N}$ or the $\alpha \mathrm{C}$ atom to nanotube, the total spin of the amino acid is transferred and is delocalized on the tube walls.

The curvature effect of the CNTs versus the interaction energy has also been studied by considering a $(5,5)$ and a $(9,0)$ tube capped with fullerene hemispheres. ${ }^{23}$ The structure of the tubes is optimized without any symmetry constraints and the geometry of the lowest minimum is presented in the inset picture of Figure 3. For both tubes, the ground state is singlet. Again, the $\mathrm{N}$-centered approach to the tube is scanned to obtain the potential energy curve. The results are presented in Figure 3. Both capped tubes have smaller binding energies ${ }^{24}$ compared to the $(4,4)$ and $(8,0)$ CNTs. Moreover, for both reactions an energy barrier of $4.5 \mathrm{kcal} / \mathrm{mol}$ is estimated. Our results are in 
agreement with previous works, which deal with the addition of small molecules to the tube walls, such as ammonia, amidogen and nitrogen dioxide. ${ }^{21,25,26}$ It is found that the binding energy is reduced and the energy barrier for bond formation increases as the diameter of the tube increases. This is a consequence of the increase of the $\mathrm{sp}^{3}$ character of the carbon atom with curvature. Further calculations have shown that the binding energy varies from -21.1 to $+11 \mathrm{kcal} / \mathrm{mol}$ between the two extreme curvature models, $\mathrm{C}_{60}$ and graphite.

Summarizing, we have performed density functional theory calculations on the structural properties of CNTs upon adsorption of various Glycine radicals. Among these clusters, the $\mathrm{N}$-centered ones have binding energies of $-16.9(4,4),-20.2$ $(8,0),-7.2(5,5)$ and $-8.2(9,0) \mathrm{kcal} / \mathrm{mol}$. In contrast, the C-centered radicals can be bound to the tube walls with endothermic reactions. The adsorption potential curves of the $\mathrm{N}$-centered radicals also show a second minimum. This minimum corresponds to physisorption and is estimated to be approximately $1 \mathrm{kcal} / \mathrm{mol}$ for all tube cases.

Acknowledgment. The present work is supported by the Greek Ministry of Education and European Union through the postgraduate program, "Applied Molecular Spectroscopy", as well as the grants IRAKLITOS and PYTHAGORAS II. We also thank the Institute of Physical and Theoretical Chemistry of the University of Bonn for making the TURBOMOLE program available.

\section{References and Notes}

(1) Iijima, S. Nature 1991, 354, 56.

(2) Terrones, M.; Hsu, W. K.; Kroto, H. W.; Walton, D. R. M. Top. Curr. Chem. 1999, 199, 189.

(3) Dresselhaus, M. S.; Dresselhaus, G.; Eklund, P. C. Science of Fullerenes and Carbon Nanotubes; Academic Press: New York, 1996.

(4) Tsang, S. C.; Chen, Y. K.; Harris, P. J. F.; Green, M. L. H. Nature 1994, 372, 159 .
(5) Davis, J. J.; Green, M. L. H.; Hill, O. A. H.; Leung, Y. C.; Sadler, P. J.; Sloan, J.; Xavier, A. V.; Tsang, S. C. Inorg. Chim. Acta 1998, 272, 261.

(6) Balavoine, F.; Schultz, P.; Richard, C.; Mallouh, V.; Ebbesen, T. W.; Mioskowski, C. Angew. Chem., Int. Ed. 1999, 38, 1912.

(7) Guo, Z.; Sadler, P. J.; Tsang, S. C. Adv. Mater. 1998, 10, 701.

(8) Bianco, A.; Prato, M. Adv. Mater. 2003, 15, 1765.

(9) Pantarotto, D.; Partidos, C., D.; Graff, R.; Hoebeke, J.; Briand, J.P.; Pratto, M.; Bianco, A. J. Am. Chem. Soc. 2003, 125, 6160.

(10) Venkatesan, N.; Yoshimitsu, J.; Ito, Y.; Shibata, N.; Takada, K. Biomaterials 2005, 26, 7154 .

(11) Panthuis, M. Chem. Biol. 2003, 10, 897.

(12) Basiuk, V. A. J. Phys. Chem. B 2003, 107, 8890.

(13) Gustavsson, S.; Rosén, A.; Bolton, K. Nano Lett. 2003, 3, 265.

(14) Huang, Y.; Guler, L.; Heidbrink, J.; Kenttämaa, H. J. Am. Chem. Soc. 2005, 127, 3973.

(15) Bonifacic, M.; Stefanic, I.; Hug, G. L.; Armstrong, D., A.; Asmus, K.-D. J. Am. Chem. Soc. 1998, 120, 9930.

(16) Wang, Y.; Iqbal, Z.; Malhotra, S. V. Chem. Phys. Lett. 2005, 402, 96.

(17) Ahlrichs, R.; Bär, M.; Häser, M.; Horn, H.; Kölmel, C. Chem. Phys. Lett. 1989, 162, 165.

(18) Eichkorn, K.; Treutler, O.; Öhm, H.; Häser, M.; Ahlrichs, R. Chem. Phys. Lett. 1995, 242, 652.

(19) Yu, D.; Rauk, A.; Armstrong, D. A. J. Am. Chem. Soc. 1995, 117, 1789.

(20) Radovic, L. R.; Bockrath, B. J. Am. Chem. Soc. 2005, 127, 5917.

(21) Zhao, M.; Xia, Y.; Lewis, J. P.; Mei, L. J. Phys. Chem. B 2004, $108,9599$.

(22) Zhao, J.; Buldum, A.; Han, J.; Lu, J. P. Nanotechnology 2002, 13 195.

(23) We have studied capped $(5,5)$ and $(9,0)$ nanotubes, because the fullerene hemispheres are capped to the tubes with a unique way (see $\mathrm{p}$ 759 of ref 3 ). Furthermore, the ground electronic state remains singlet even for the zigzag tube as our calculations have shown.

(24) Closing the tubes with $\mathrm{C}_{60}$ hemispheres has a very little effect on the binding energies. Examining both models, capped and open hydrogen ended $(5,5)$ CNTs gave an energy difference less than $0.5 \mathrm{kcal} / \mathrm{mol}$.

(25) Zhao, M.; Xia, Y.; Ma, Y.; Ying, M.; Liu, X.; Mei, L. Phys. Rev. B 2002, 66, 155403.

(26) Ruffieux, P.; Gröning, O.; Bielmann, M.; Mauron, P.; Schlapbach, L.; Gröning, P. Phys. Rev. B 2002, 66, 245416. 